rather than a boil, as the patient had described. I told him my suspicions, gave nim directions to completely empty the bowels, and confine himself to liquid diet. Three days after I gave him a careful examination under an anesthetic, having made all the necessary preparations to operate. I found a small ulcer just below the internal sphincter, and readily discovered, by the aid of a probe, that it gave rise to a sinus, which I freely opened into the bowel in the usual manner, thoroughly curetted the abscess cavity and the fistulous tract, carefully washed it out with pure hydrogen dioxid, and, commencing just above the superior border of the ulcer in the bowel, with large-sized catgut carefully stitched the incised parts together with continuous suture, carrying the sutures sufficiently deep to incorporate all of the parts separated as it came to the external opening. With the aid of a speculum I packed the bowel with strips of iodoform gauze, constipated the patient by the use of opium, and for five days allowed nothing but concentrated liquid food and a small quantity of acidulated water. The sixth day the first suture beyond the anus separated, and careful examination showed that the parts were healed by first intention. I removed the gauze and thoroughly cleared out the bowel by the aid of Husband's magnesia and hot lemonade, which, by the way, I have found the most satisfactory of all forms of purge for all abdominal operations, as it invariably gives thoroughly liquefied stools from the very first. At the end of the sixteenth day this patient was out of bed, and in three wreeks was dressed and out of doors.

419 Boylston Street.

\section{ON THE RELATION OF DISEASES OF THE ACCESSORY CAVITIES TO DISEASES OF THE EYE.*}

\section{BY J. H. ЗKYAN, M.D.}

WASHINGTON. D. $\mathrm{C}$.

The relation between the diseases affecting the accessory sinuses of the nose and those of the orbit and its contents is such an intimate one that it seems strange so little has been done in the past in the way of clearing up the hazy ideas that have hitherto existed as to the influence of diseases of these pneumatic spaces on diseases of the eye. Indeed, in the ophthalmologic handbooks and text-books of to-day, very little on the relation of orbital diseases to the suppurative affections of the accessory cavities has been written, authors contenting themselves with a brief mention of some of the more decided orbital complications which have their origin in the pnenmatic cavities.

'This is the first time in America, I believe, that a joint meeting between the rhinologists and ophthalmologists has been held with the view of jointly studying these oftentimes very obscure and serious affections. I feel such a discussion can only be followed by the best results, for there is no region where so much depends on the harmonious action of the two specialties in order that the patient may be reliered from intense suffering, an eye preserved, or a life saved.

It is probably a matter of assurance to many of us that, if a better understanding of these diseases had only existed, cases of so-called supraorbital and infraorbital, or optic neuralgia would not have been treated so long without benefit, some of which have gone to the verge of excision, only to find the nerves were not the seat of the

*Presented to the Section on Ophthalmology, at the Fiftieth Annual Meeting of the American Medical Association, held at Cofumbus, Ohio, June 6-9, 1899. disturbance, but that one of the cavities adjoining the nose was the source of the trouble.

The eye, partially surrounded by the accessory cavities whose thin walls go to make up the walls of the orbit, is very sensitive to any pathologic condition that takes place within them, and presents symptoms in many instances which, when carefully considered, will enable the surgeon to definitely locate the source of the disease. It is my custom to submit all such cases, no matter how trivial they may at first appear, to a most searching ophthalmologic examination. In this way I have been able to locate some obscure affections of the accessory sinuses, which would have been most difficult to recognize by rhinoscopic examination alone.

It is to Dr. W. H. Wilmer and his assistant, Dr. Greene, who have made most of the ophthalmologic examinations of my cases, my thanks are due for the painstaking care which they have practiced in making the searching examinations that such cases require.

Several of the cases I propose to briefly mention in this paper have been previously reported to the American Laryngological Association, but short abstracts will here be made of them in order to illustrate some of the points I desire to elucidate.

As in diseases of the nose, the affections of the frontal sinuses are frequently accompanied by ocular reflex disturbances; but they are frequently overlooked beeause of the difficulty in making a diagnosis of the diseased condition of the sinus. The reflex symptom most frequently observed in diseases of the frontal sinus is above all neuralgia of the supraorbital nerve, and it is present in both the acute and chronic forms of inflammation affecting the cavity. It occasionally happens that this symptom has been present long before the characteristic secretion makes its appearance in the nose. This neuralgia is not always constant but frequently occurs at regular intervals, and it does not always correspond to the diseased area, but may radiate to the parietal and temporal regions of the affected side. I have also occasionally noticed it to extend to the opposite side. This is probably one of the most frequently misinterpreted symptoms, puzzling not only the general practitioner but the specialist also. When we take into consideration, however, in the majority of cases of frontal headache either constant or intermittent in character, that there are other evidences of sinus disease, such as pain on pressure above and below the supraorbital ridge, especially at the inner angle, accompanied by a unilateral discharge of mucus or pus from the corresponding nostril, it seems difficult to understand why its true signifcance should not be more frequently understood.

The frontal sinuses vary in size to a great degree in different individuals. After an examination of 120 skulls, Herbert Tilley found the sinus large enough in some instances to contain an ordinary bean, while in others it was ten times as large. He considers a sinus normal where it measures $28 \mathrm{~mm}$. from the median line ontward, and in the vertical extent measured from the nasion from 20 to $22 \mathrm{~mm}$. The size of this cavity, where the seat of a catarrhal or suppurative inflammation which is the most frequent pathologic condition met with, has a very important bearing on the ocular symptoms; much depends also on the depth of its floor, and on the thickness or thinness of its walls.

The secondary changes in the orbit, due to frontal sinusitis, are of the greatest interest. and they may bo brought about simply by mechanical pressure, resulting in change of form of the orbit, in a displacement of the 
eyeball with a disturbance of its mobility and function, and in an interference with the flow of tears.

Disturbances in the function of the eye of a high degree have been observed in cases of frontal sinusitis, such as dilatation of the pupil and congestion of the papilla, varying in intensity with the degree of pressure; a narrowing of the field of vision, especially for colors, scotomata, and in very severe cases amblyopia or amaurosis. This, according to Kuhnt, serves to bring into prominence the fact that the peripheral and central sight in these cases can be raised up to a certain grade through intense illumination which points not to atrophy, but only to a lowering of the perception and conductibility. Frequently after the removal of the mechanical obstruction caused by the abscess, there is a rising of the central and peripheral sight; changes in refraction toward the hypermetropic and astigmatic side are observed in the cases where the pressure of the dilated sinus wall is made at the equator of the eyeball or on its posterior half. If the purulent secretion is not discharged through the nose, it may result in a diseased condition of the osseous walls of the sinus, causing an ostitis or

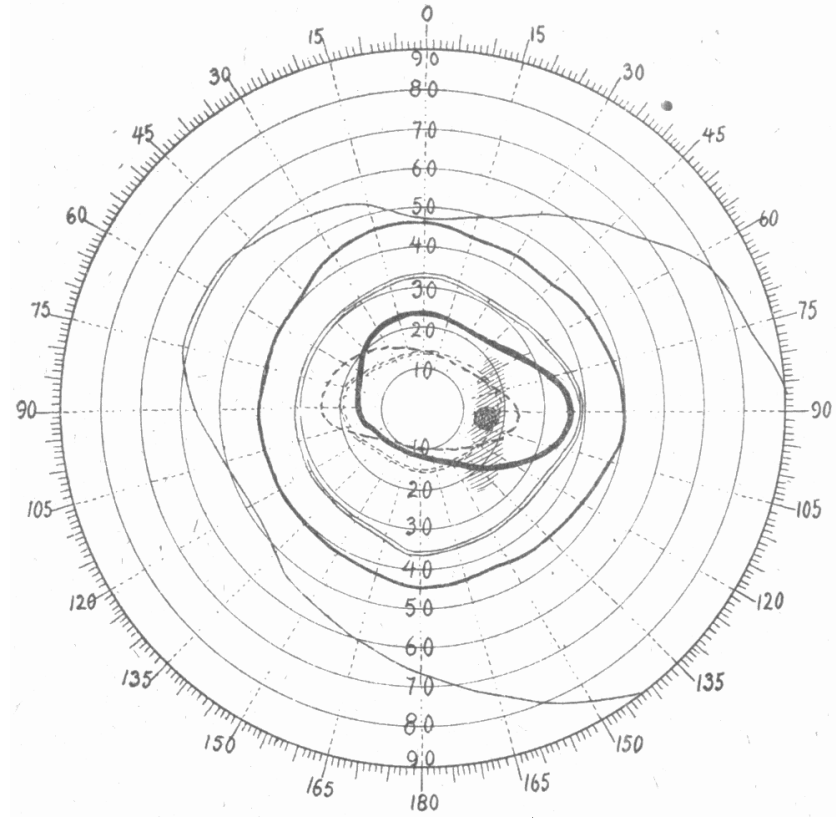

Chart 1 - white $;==$ black; - . - yellow; red; - a = green

periostitis of the walls of the orbit, the walls finally becoming so thin that the secretion can be discharged through a fistulous opening in the supraorbital ridge, through a fistulous opening at the inner angle of the orbit where the floor of the sinus is the thinnest, or farther back into the orbit, producing an orbital abscess which is accompanied by all the compression phenomena from the side of the eye.

Abscess of the orbit is rarely primary. In the majority of instances it is the result of an extension of an infiammation from one of the adjoining sinuses, generally the frontal. The first symptom that shows itself may be the appearance of a small swelling at the inner angle of the orbit, while at other times the phenomena of ocular compression make their appearance, such as lachrymation, pain in the eve, exophthalmus, strabismus, mechanical diplopia, edema with a stretching of the upyer lid and, when the disease has advanced to any extent, ectropion. Before the pus has made its outward appearance there may be considerable chemosis which partly or completely immobilizes the eyeball, especially for its upward or downward movements.
The following is a brief abstract of a case of orbital abscess referred to me by Dr. Belt of Washington: CHRONIC FRONTAL SINUSITIS, CARIES OF THE FRONTOETHMOIDAL CELLS, ORBITAL ABSCESS, FISTULOUS OPENING AT THE INNER ANGLE OF THE ORBIT.

CASE 1.-On May 2, 1897, this patient consulted Dr. Belt, after an attack of influenza, for a severe pain in the left eye. At this time the left upper and lower lids were red and swollen, and there was considerable chemosis of the conjunctiva. The eyeball was immovable, owing to the great swelling of the orbital tissues. The swelling increasing, an incision was made at the inner canthus and a large quantity of pus evacuated. A probe introduced into the wound was found to pass into the frontal sinus through a small opening at the inner angle of the orbit. Owing to the very anemic condition of the patient, further operative measures were not deemed advisable until his general health improved.

When I first saw him, Sept. 10, 189\%, there was a small fistulous opening at the inner angle of the left orbit, through which pus in considerable quantities was dis-

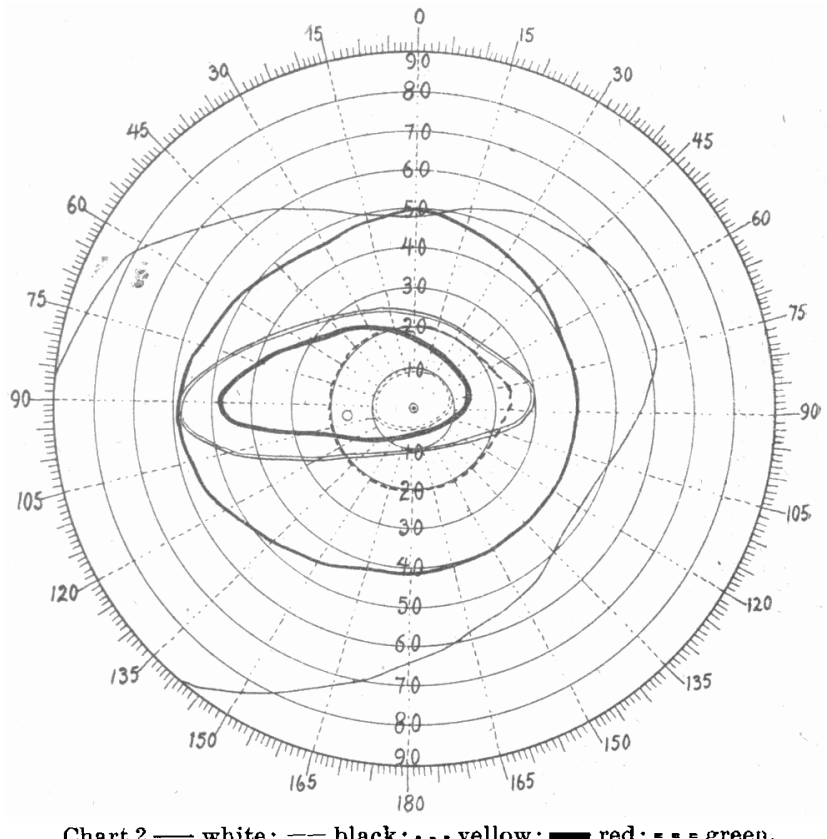

Chart $2 \longrightarrow$ white $;==$ black: $\cdots$ yellow; - red $;==$ = green.

charging. There was a slight drooping of the upper lid, but no swelling of the skin over the frontal sinus, and no pain on pressure.

'The patient was admitted to the Episcopal Eye, Ear and Throat Hospital, September 17, when after shaving the eyebrow and thoroughly cleansing the parts, an incision was made along the eyebrow, commencing just within the supraorbital notch and extending to the riasal boss. After elevating the skin and periosteum, a small opening in the orbital plate of the frontal bone was found, communicating with the frontal sinus. This cipening was sufficiently enlarged by means of a gouge and rongeur, to admit of a thorough examination of the cavity, which was found filled with granulation tissue, and also found to be the seat of caries at its junction with the fronto-ethmoidal cells. The sinus was thoroughly curetted, and all granulation and carious tissue was removed. The frontonasal duct being closed, arr opening into the nose was made with a trocar, and a selfretaining drainage-tube passed into the nose. The cavity was irrigated with a solution of bichlorid of mercury, $1-3000$, the wound closed and hermetically sealed with 
iodoform and collodion. The sinus was irrigated daily throngh the drainage-tube, with a saturated solution of boric acid. At the end of a week, the external wound having healed, the patient was discharged from the hospital, and in ten days, there being no secretions from the sinus, the drainage-tube was removed. This is the form of orbital abscess so frequently met with resulting from a suppurative inflammetion in the frontal sinus. It is, however, not always inevitable for a perforation of the walls of the sinus to take place, for in certain cases, in spite of the most minute examinations, we do not find any perforations. Pauas has described such cases as abces circonvoisin. He belicves, on account of the thin walls of the sinus, the process is propagated to the periosteum without any perforation ${ }^{1}$.

Points valuable in the diagnosis of orbital abscess resulting from suppurating frontal sinusitis, are the position of the eye, the direction in which the eyeball is pushed, as well as the corresponding immobility of the eye. If the tumor is felt above, or above and internal, and if the eyeball is pushed downward and a little outward, while its movements upward and inward are

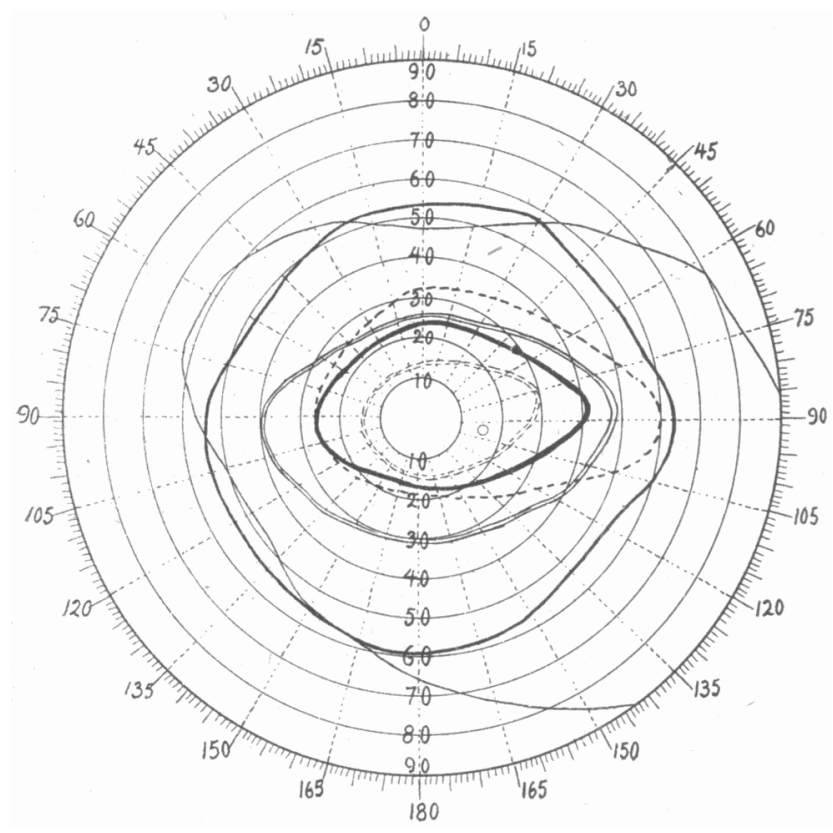

Chart 3 - white ; == black; $\ldots$. yellow ; - red ; - . - green.

diminished, it indicates a breaking through from the frontal sinus.

A suppurative inflammation of the frontal sinus, of any severity or duration, in my experience, rarely exists alone, but is usually accompanied by caries of the frontoethmoidal and ethmoidal cells. While this complication occasionally gives no evidence of its existence by a rhinoscopic examination, it is much in evidence as soon as the frontal sinus is opened. In such cases the ocular symptoms are increased in severity and render the diagnosis more difficult, for, added to the signs of frontal sinus inflammation are those which are peculiar to the ethmoidal alone. In such cases $I$ have found the visual fields for colors always narrowed, exophthalmus more pronounced than in simple uncomplicated frontal sinusitis, especially so when the lateral and superior pressure is either at or posterior to the equator of the eyeball - the displacement is pronouncedly downward and outward, and its movement inward especially restricted.

1 Progrès Med,, 1890.
CHRONIC FRONIAT, SUPPURATLNG SINUSITIS AND SUPPURATING ETHMOIDITIS.

CASE 2.- This is a very instructive one and illustrates the various disturbances these serious inflammations have on the eye. S. G., aged 15 , was referred to me Oct. 26,1898 , by Dr. Wilmer. The patient stated that, while never robust, he had enjoyed fairly good health. He does not recall having had infiuenza.

During August of the present year, while at the seashore, he was taken ill with what was supposed to be malaria, but which finally proved to be an inflammation of the right frontal sinus. During this illness, in the absence of Dr. Wilmer from the city, he was seen by Dr. Burnett, who kindly gave me his notes of the case at this time:

August 12, there was an enlargement, very hard to the touch, near the right lachrymal region, but superior to the sac, without discharge from the puncta on pressure on the sac. The nasal duct was patulous, and fluid passed into the nose on syringing.

August 14, he had a chill followed by fever; the right upper lid red and edematous, the swelling extending to the forehead at the root of the nose where it was more tender than else-

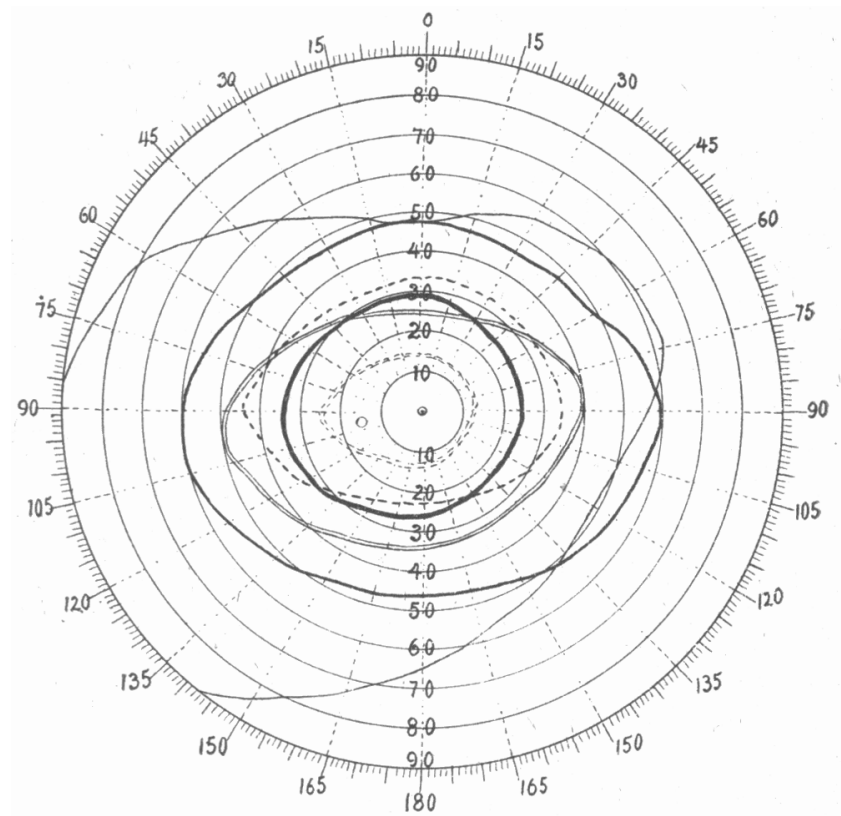

Chart 4 - white ; $:=-$ black ; - . - yollow ; - red ; - . - green.

where; quite a marked exophthalmus and diminished movement of the right eye; pupil larger than the left.

August 15, swelling was slightly diminished; no chemosis of the conjunctiva; temperature normal.

August 17, some subsidence of the swelling and exophthalmus was noted; pupil normal; eye more movable; swelling confined to the upper lid; the fundus showed no change when examined with the ophthalmoscope; some bloody mucus discharged from the right nostril and increased by pressure on the tumor at the inner canthus.

August 20, the swelling was gone, no dipiopia in any part of the field of fixation, no defect in the visual field on rough testing.

August 22, there was still some swelling at the inner canthus and tender on deep pressure.

About this time the patient left the city and did not return until Oetober 26, when I saw him. He then complained of intense frontal headaches, pain on pressure above and below the supraorbital ridge, and over the swelling at the inner angle of the orbit; slight exophthalmus. He complained of chilly sensations, giddiness and nausea, temperature 100 . He was quite anemic in appearance, and stated that he had lost twenty pounds during the preceding month. 
Rhinoscopic examination showed the right middle turbinate much swollen and, on probing, caries of the cthmoid cells was discovered. The secretions were purulent and, while not very profuse, were observed to come principally from the right nostril, also flowing back into the postnasal space over the posterior end of the middle turbinate body. The electric light showed the right frontal sinus opaque, while the left frontal cavity and both maxillary sinuses were translucent.

Opthalmologic examination, made by Dr. Wilmer on October $2 \%$, was as follows:

Ophthalmometer showed a corneal astigmatism in the right eye, of $0.50 \mathrm{D}$., with rule, in the left eye corneal astigmatism of $1.50 \mathrm{D}$.

R. E. V. $=20 / 15$; accepted cyl. +0.25 ; axis 180

L. W. V $=20 / 30$ with eyl. $+0.62,90=20 / 15$.

Reads Taeger No. $12^{\prime \prime}$ equal; 20 feet, Exo. $2^{\circ}$ ad. $4^{\circ}$, ab. $4^{\circ}$. Color sense was good. The ophthalinoseope showed no change at all in the fundus. The perimeter showed a decided narrowing of the visual fields for white and colors in the right eye. There was also marked scotoma with its center corresponding to the normal blind spot, amounting to something more than its mere enlargement-as shown in the charts 1 and 2 .

The left frontal sinus was the seat of a slight mucocele which was sufficient to reduce the visual field for white and colors.

'The patient was admitted to the Episcopal Eye, Ear and 'Throat Hospital, October 2\%, and on the following day, under ether anesthesia the frontal sinus was opened in the following manner: After shaving the right eyebrow and thoroughly cleansing the parts, an incision was made through the brow commencing just within the supraorbital notch, and continued on to the nasal boss.

The flap, composed of integument and periosteum, was elevated, and a button of bone $1 \mathrm{~cm}$. in diameter was removed by means of a crown trephine applied just above the supraorbital ridge and about one line from the median line. The outer table showed signs of commencing softening: On opening the sinus it was found filled with thick fetid pus and granulation tissue. The anterior wall and fronto-ethmoidal cells were in a carious condition, as were also the ethmoid cells, at least as far back as the junction of the middle with the posterior cells.

All diseased tissue was removed from the sinus, and with the litile finger in the nose for a guide, all the ethmoid cells were removed with a sharp curette, as far back as they seemed to be diseased; a large opening was thus left between the sinus and nose. The walls of the sinus were then touched with a 20 per cent. solution of the chlorid of zinc, the cavity packed with iodoform gauze, ons end being drawn out through the nose; the wound was then closed and hermetically sealed with iodoform and collodion.

On the second day of the operation the gauze drain was removed from the sinus through the nose, and the cavity irrigated daily with a saturated solution of boric acid. The patient made a rapid convalescence, and was discharged from the hospital on November 9 , the secretions very much diminished in quantity. The irrigations were continued daily for two weeks longer, when the secretions from the sinus practically ceased. Examination of the eve, made nineteen days after the operation, showed no exophthalmus; mobility of the eye, normal.

R. E. V. $=20 / 20$;

L. E. V. $=20 / 20$;

$20 \mathrm{ft}$., exo. $1 / 2$, ad. $12^{\circ}$, ab. $4^{\circ}$.

The ophthalmometer showed a corneal astigmatism of $1.50 \mathrm{D}$. with the rule in both.

The visual fields, taken November 16, showed a marked improvement for white and colors, although they did not reach quite the normal standard, as will be observed by referring to Charts 3 and 4 . When these abscesses have existed some time, they may result in a disease of the deeper structures of the eye, as in the form of an iridochoroiditis.

\section{CHRONIC ABSOESS OF THE FRONTAT SINUS AND ETHMOID} CELLS FOLLOWED BY A IRIDOCHOROIDITIS.

CASE 3.-This case demonstrates the serious results that are apt to follow these suppurative conditions. Mr. - - , aged 71 , consulted me Dec. 4, 1894, giving a history of frontal neuralgia of ten years' standing, the pain starting at the inner angle of the orbit, radiating over the corresponding side of the head and face, and increased in intensity by an acute coryza. For a number of years he has had a yellowish purulent discharge from the left nostril, no swelling at the inner angle of the orbit, and he states that he has no disturbance of vision.

Rhinoscopic examination showed the left middle turbinate slightly enlarged, and a thin purulent secretion was observed coming from above, and below it in the middle meatus. The probe, when passed into the middle meatus, revealed an extensive caries of the ethmoid cells. By means of a sharp curette I removed large quanlities of pus and carious tissue from the region of the anterior ethmoid cells, after which the probe could readily be passed into the frontal sinus. The cavity was frequently irrigated with a saturated solution of boric acid. As this was followed by a very decided improvement in his condition, it was thought best not to resort to any more radical measures for his relief on account of his age and a decidedly irregular heart action.

From time to time $I$ have scen this patient, and about two years ago there seemed to be a tendency to an extension of the suppuration process backward into deeper and posterior ethmoid cells. Drainage, established by means of an occasional curetting, seemed to give the patient relief from his painful symptoms.

About two years ago the left eye commenced to trouble him, and he then consulted Dr. Herbert Harlan of Baltimore who has kindly given me the following notes on his eve-condition since he has been under his observation:

February 11: He complained of "a litthe mistiness of the left eye." V. O. D. $=15 / 100$, V. O. S. $=8 / 100$. He had been wearing bifocals. +1.50 gives $O$. D. $15 / 30 ; 0$. $\mathrm{S} .15 / 200$. The right fundus was a little cloudy, but good. Deep physiologic pitting of disc was evident. The left had large floating bodies in the vitreous. The vitreous was muddy. A patch of choroiditis was made out to the nasal side. He was put on a tablet containing iron. arsenic and strychnia, and his reading glasses changed to $+4 \mathrm{D}$.

February 25; The eye was a little better. V. O. S. $=$ 15/100 with glasses. Treatment was changed to ferri ot hyd. chlor. cor. comp. (Mulford).

April 7: "Eye doing well until a few days ago." Now had marked iritis with adhesions. Atropia solution was used.

April 10: The pupil was partly dilated, the fundus muddy, and the eye red. Treatment was kal. iod., grs. $x v$, in essence of pepsin t. i. d.

April 28: "Eye more inflamed, last two days. Very uncomfortable. No great pain." 'Tension +, blood in ant. chamber, cornea cloudy. Eserin was instilled, and after a few minutes the eye felt better, and tension was less. Eserin was ordered and $K \mathrm{~K}$ stopped, but ferri et hyd. given.

May 3: Tension was less and less olond in the anterior chamber

June 17: "Eye did well until about ten days ago." Now very red, with blood in the anterior chamber, the pupil was closely contracted, tension $+\mathrm{I}$. Atropia was ordered to be used carefully.

June 21: The left eye was better, the blood in the anterior chamber nearly absorbed, vitreous muddy; no fundus; light only. 
July 17 ; O. S., no longer red; cornea hazy; no fundus; O. D. $+2.50,15 / 15 ; \mathrm{B} .+4.50$ reading.

April 18: O. S.; cornea clear, lens opaque, light; O. D. pupil a little dilated, and responds sluggishly to light. $\mathrm{V}$.= $15 / 15$; field taken and found good; use of his distance glasses advised. Fraser's quin. comp. tablets were ordered.

May 18: The condition was the same.

November 15: The condition was the same.

December 30 : A letter says the left eye is red and irritable and he wishes some other drops.

Jan. 26, 1899: +0. S., red and irritable; pupil closed; no light; O.D. well; kal, iod. and Fairchild's essence of pepsin were ordered.

March 20: O. S., red and heavy; tension; atropia was ordered and KI continued.

April 13: Enucleation was advised for 0 . S.

ITIMMOIDAI, AFFECTIONS IN RELATION TO DISEASES OF THE TXE.

Affections of the ethmoid cells are generally caused by diseases of the nose, and may exist alone, or complicate suppurative inflammations in the frontal, as has been previously mentioned, also in the maxillary and sphenoidal sinuses. According to the intensity of the inflammation and the cells involved, caries of the ethmoid may or may not be accompanied by ocular symptoms, a great deal depending whether the abscess is open or closed, and whather the anterior or middle and posterior cells are involved. In the open variety, where the pus has a free discharge into the nose, the eye may escape any injurious effects until the carious process has made sufficient advances to involve the deeper cells.

In the closed variety, however, the ocular symptoms are almost immediately present. Abscesses in this region are accompanied by the same vcular disturbances that are met with in frontal sinus inflammation, the pressure symptoms varying somewhat from those in the upper cavity. Supra- and infraorbital neuralgia, socalled cysts of the orbit, orbital abscess, exophthalinus, disturbances in refraction, scotomata, iridochoroiditis, changes in the visual field, are the symptoms most frequently found in well-advanced cases. The so-called cysts of the orbit are generally the manifestation of some form of disease of the ethmoid cells, either in the form of a closed abscess or a dilatation of the cells, the pressure being so great as to cause a marked displacement of the eye.

If the middle or posterior cells are affected without any involvement of the other neighboring cavities, the displacement of the eye is generally outward, while the inward mobility of the eyeball is decidedly interfered with.

At times, when there is a swelling and projection of the cells toward the orbit, it becomes very difficult to differentiate between a closed abscess, a cyst or a solid tumor.

The following case illustrates some of the difficulties that are met with:

NASAL POLYPT, ALVEOLAR SARCOMA OF THE ETHMOID CELIS, LATLE INVOLVING THE ORBIT; PURULENT ACCUMULATION IN THE ANTRUM.

C.1SE 4.-Mrs. — Dr. Stone, U. S. N., and stated that in October, 1898 , she noticed a great difficulty in breathing through the left nostril, which on examination was found to be due to the presence of several large mucous polypi. These were removed in January, 1899, and some time afterward she had a profuse hemorrhage from that side of the nose which, however, was not attributed to the removal of the polypi.

March 1, when she first consulted me, she complained of pain in the supraorbital and infraorbital regions, pain in the eye, inability to breathe through the left nostril, photophobia and lachrymation. Examination ef the eye, by Dr. Wilmer, showed both lids of the left cye swollen, there being also an extensive chemosis of the conjunctiva at the lower inner angle of the eye. Motion of the eyeball was interfered with to some extent, owing to the swollen condition of the orbital tissues. On palpation a firm mass was found along the orbital ridge of the malar bone, extending from the inner canthus 5 mm. outward.

No ophthalmometric reading could be made, owing to the condition of the eye. The patient was myopic, but normal sight could be obtained by use of proper lenses. Examination of the muscles showed the following: image seen with left eye, $11 / 2$ to the left and $1^{\circ}$ below that of the right. Ophthalmoscopic examination revealed the veins and arteries very much engorged, slight pulsation, and slight elevation of the retina at the inner lower angle.

'The day following, the chemosis had increased greatly and examination showed continued diplopia. The fields for white and colors were normal. Rhinoscopic examination showed the left nostril partially occluded by numerous small mucous polypi springing from a greatly swollen middle turbinate body. No pus was observed in the nasal chamber, but on forcibly blowing the nose small quantities of thick purulent secretion could be blown out, and she complained of a constant dropping into the postnasal space. Fxamination with the electric light showed both frontal and right maxillary sinuses translucent, while the left was absolutely opaque. The diagnosis in this case lay between a closed abscess of the ethmoid cells bulging into the orbit, and secondarily affecting the maxillary sinus, a dilatation of the ethmoid cells, or a solid tumor originating within them. The patient having lost her teeth several years before, on March 2, I perforated the left antrum, through the alveolar ridge about the location of the first molar, by means of a small trephine, and evacuated about a dram cf rather thick pus. The cavity was irrigated daily with a saturated solution of boric acid, with the result of an almost complete disappearance of pus within three or four days.

I made several attempts to reach the seat of the ethmoid disturbance through the nose by means of a curette, but without much success; as the chemosis was rapidly increasing and the eye symptoms becoming more threatening it was decided to operate without further delay.

March 26 the patient was etherized, and, assisted by Drs. Wilmer and Greene, an incision was made through the base of the lower lid extending from the inner angle to nearly the full extent of the infraorbital ridge; the bulging ethmoid cells were exposed, and opened by means of a perforator. Through this opening a curette was passed into the ethmoid cells and with the little finger in the nose for a guide I was enabled to remove nearly all of these cells, which were found to be broken down and filled with pus and granulation tissue as far back as the posterior cells. The nasal wall of the antrum in relation with the ethmoid bone was also found badly diseased, and was removed, leaving a large communication between that sinus and the nose. The parts were then thoroughly cleansed with a boric acid solution, and the external wound closed. There was no microscopic examination made of the tissue removed from the ethmoid cells at this time. The external wound healed quickly, but at the end of a week it broke down at its inner third; the nose and antrum were irrigated daily with a boric acid solution. 
"The patient's condition was much improved for a time by the operation, especially in the subsidence of the chemosis and the pressure on the eye. The secretions from the nose and from the external wound were at no time profuse.

About two weeks after the operation she complained of increasing pain in the eye, in the infraorbital and supraorbital and temporal regions, the eye condition remaining about the samc.

April 12 the eye condition was not so good, there being increased swelling of the lower lid, very hard to the touch, chemosis increasing. The nose, which had been thoroughly relieved by the first operation, now showed a return of the swelling, which on examination was seen to comc from the seat of the operation.

By April 14 the pressure on the eye had increased decidedly - the preceding two days - chemosis being very much increased. 'The symptoms were so threatening that a second operation was insisted on.

April 15 the patient was etherized, and Dr. Wilmer, operating through the previous incision in the lower lid, discovered the floor of the orbit filled with a tumor formation of very firm consistency. It had extended from the ethmoid cells along the floor of the orbit, infiltrated the lower lid and was extending into the upper lid back of the lachrymal sac.

Not having authority to remove the eyeball and the contents of the orbit, as the indications so strongly called for, he had to be content with removing as much of the tumor as possible through the external wound, removing a portion of the floor of the orbit at its juncture with the ethmoid bone. A drainage-tube was passed into the orbit, and the wound closed. A microscopic examination, made by Dr. Walter Read, U. S. A., showed the tumor to be of the alveolar sarcoma variety. The patient's husband, who is a physician, realizing the unfavorable prognosis of the case declined a more radical operation for her relief.

April 20 the patient was discharged from the hospital, in order that she might be removed to her home in New Xork State. In a letter shortly after her arrival home her huslsand stated that the growth was slowly increasing.

DISEASES OF 'THE SPIHENOLDAI, SINUSES IN RELATION TO DISEASES OF THE EYE.

'There is no means of knowing to what extent sphenoidal disease exists. Whether it is as frequent or less frequent than inflammations in the neighboring cavities of the nose is a question yet to be determined. In cases in which the symptoms are not well defined, it is impossible to make a diagnosis, and it is frequently only when the disease has advanced to such an extent as to cause serious eye disturbance or other symptoms that threaten an extension to the meninges, that we can form a definite opinion as to the condition of these cavities. These cavities are subject to the same forms of inflammation noticed in other sinuses, and they are also occasionally the seat of tumor formations. A suppuritive inflammation in these cavities alone is exceedingly rare, for it is generally associated with disease of the ethmoid and frontal sinuses.

The diagnosis of ahscess of the sphenoidal sinuses rests almost entirely on rhinoscopic and ophthalmologic examinations, for the subjective symptoms may be wholly wanting, or of such a manifold variety as to make it impossible to diagnose with even a slight probability a disease of this cavity. The most common rhinoscopic finding is the appearance of pus in the superior meatus, posteriorly. Even this is not positive evidence that the source of the trouble is the sphenoid alone, for in many instances the posterior ethmoid cells are the seat of the disease. But when these cells are the seat of a suppurative inflammation, it is safe to say the sphenoidal cavities are also involved, for the bony partition that separates these cavities is so thin they can not withstand any pressure without giving way under it, thus forming a direct communication for the extension of any pathologic process from one cavity to the other. Occasionally the anterior wall of the sphenoid sinus may bulge forward. When this occurs the source of the purulent secretion is then readily recognized.

In mild cases, where the pus finds a ready exit through the nose, the eye may not be involved; but where the infiammation is severe from its outset, or there is an obstruction to the outflow of pus, pain in the occipital region, extending to the vertex, will be marked. There may be also an increased lachrymation, photophobia, pain in the orbit back of the eye, exophthalmus, ptosis, strabismus, diplopia, orbital abscess, and from a partial to a complete loss of vision.

The following case represents some of the manifestations of this disease.

ABSCESS OF THE SPHENOID SINUS, PARALYSIS OF THE EXTEIINAL RECTI.

Case 5.-Mr. - aged 5\%, was referred to me by Dr. Wilmer, April 17, 1899. When I first saw this patient he complained of intense pains in the occipital region, radiating to the rertex; giddiness, nausea, and double vision. There was a profuse fetid purulent discharge from both nostrils and from the postnasal space. After thoroughly irrigating the nose, a rhinoscopic examination showed the frontal, ethmoidal and maxillary sinuses to be free from discase. The posterior wall of the nasopharynx was observed bulging forward, nearly touching the velum palati, the bulging being greater on the right than on the left side. Pus in large quantities was seen in the vault of this space, flowing forward in the nasal chambers and below into the pharynx.

He was first seen by Dr. Wilmer, Feb. 27, 1899, when he showed a complete paralysis of the right external rectus only. Examination of the eye, with correction for refraction showed the sight normal in each eye. Pupillary reaction was normal, as were also the visual fields for white and for colors. The interior of the eye showed nothing abnormal.

April 17 there was a paralysis of both external recti. but no other changes in the eyes. He complained of great pain at the back of the head and vertex.

April 21 the patient was admitted to the Episcopal Eye, Ear and Throat Hospital, and on the following day, under cocain anesthesia, I. opened the left sphenoidal sinus by means of a gouge passed through the nose. The opening was made rather too high, but was suffi. ciently large to admit of a free discharge of pus, which gave him considerable relief. and which lasted for several days. At this time I supposed both sinuses communicated, and hoped that one opening would be sufficient, but the pains soon returned and seemed to increase in intensity.

April 28, under ether anesthesia, I made a free opening in the right sinus, by means of the gouge passed throngh the nose, using my finger in the postnasal space as a guide. This was followed by a profuse discharge of very thick fetid pus. The sinus was freely curetted, removing as much carious bone as could be reached by my instrument, and was irrigated with a saturated solution of boric acid. This operation was followed by almost complete relief from pain, and a very decided 
improvement in the paralysis of the right external rectus, while there is none in the paralysis of the left.

The sinuses have been irrigated daily with a boric acid solution, resulting in a decided dimunition in the quantity of purulent secretion. He was discharged from the hospital on Hay 2, greatly improved. 'The opening in the left sinus shows a tendency to close, and in consequence the occipital and vertex pains have returned. but they are not so intense. This cavity will probably have to be opened again. This case is especially interesting from the fact that the other sinuses are not involved, and the pressure, instead of being made on the optic nerve, as is usually the case, is directed upward and outward and involves the abducens nerves just before passing through the sphenoidal fissure.

DISEASES OF THE MIXILLARY SINUS IN RELATION TO THE DISEASES OF THE EYY

The maxillary sinus is subject to the same diseased conditions that are met with in the other accessory cavities, and probably is the most frequently affected, as it has both the teeth and the nose from which pathologic processes may extend. It probably is, however, less frequently complicated with diseases of the eye than the other accessory sinuses.

Many of the eye sylnptoms that are met with in abscess of the maxillary sinus are reflex in character, such as narrowing of the field of vision, photophobia, dilatation of the pupil, blepharospasm, and ptosis; while others are the result of an inflammatory extension; such as osteoperiostitis, abscess of the orbit, dacryocystitis, and iridochoroiditis.

It will be observed that there are many symptoms of diseases of the eye, when associated with accessory sinus inflammations, which are common to one or more of the affected cavities. There are, however, certain symptoms which are peculiar to certain sinuses, and it is only by the closest observation of these cases, both from the rhinologic and ophthalmologic side, that we can hope to make an early diagnosis. While pain is a symptom met with in these affections, its location will frequently aid one in determining the cavity which is the seat of the inflammation. This, however, must not be depended on alone, for occasionally in ethmoidal abscess the pain is referred to the supraorbital ridge as well as to the eye and infraorbital region.

Orbital abscess may occur as readily from an extension of an inflammation of one cavity as from another. I believe, however, basing my opinion on my own experience, it more frequently results from frontal sinus disease. A narrowing of the field of vision for white and for colors may follow an abscess in either the frontal, ethmoidal, sphenoidal, or maxillary cavities. It is, however, more frequently met with when the frontal and ethmoidal sinuses are jointly diseased. Refractive disturbances are more frequently noticed in inflammations of the frontal and ethmoidal cavities, and generally in those cases where the pressure is exerted either at or posterior to the equator of the eyeball.

Exophthalmus may be present in any well-advanced abscess of the accessory sinuses; the extent and direction in which the eye is pressed from its normal plane indicates the cavity which is affected; e. g., in frontal disease the eye is pushed forward and downward, in ethmoidal, forward and outward, in sphenoidal, directly forward and slightly outward, while in maxillary disease it is directed upward and outward.

Sudden blindness, together with ptosis and strabismus, when associated with abundant discharge into the postnasal space, is indicative of sphenoidal disease.

\section{EYE TROUBLES ATTRIBUTABLE TO NASO. PHARYNGEAL AND AURAL DIS- TURBANCES.*}

BY JOSEPH A. WHITE, M.D.

Professor of Ophthalmology, and Associate Professor of Otology and Laryngology, University College of Medicine. RICHMOND, VA.

The direct anatomic connection between the eye and nose makes it easy to understand how disease can spread from the latter to the former by way of the nasal duct. Moreover, the intimate nervous and vascular connection between the two organs also plays an important part in the propagation of disease from the nose to the eye. Both these modes of transmission have been recognized since the last century, and referred to by different authors, but Bresgen-1881-was the first to lay any special stress on the fact that conjunctival catarrh was often dependent on nasal catarrh. Since his article appeared, medical literature has had numerous contributions on this and other eye troubles of nasal origin, whether by direct or reflex communication. Appended to my article on "Neurosis of the Nose and Nasopharynx," in Burnett's "System of Diseases of the Ear, Throat and Nose," and to Dr. Gould's article in the same treatise, on "Disease of the Eye Dependent on Diseases of the Nose," can be found an extensive bibliography of the subject from the earliest times to 1892 . Since then, other contributions on the subject have appeared from time to time, adding to the accumulation of testimony to the importance of considering the nose as an etiologic factor in eye disease.

From these referencesit will be seen that, according to \& large number of observers, we have not only troubles of the lachrymal apparatus by direct transmission of pathogenic organisms from the nose to the eye, but a large number of other eye diseases traceable to direct or reflex extension from nasal and nasopharyngeal causes, such as asthenopia, photophobia, retinal hyperesthesia, muscee volitantes, ocular neuralgia, contraction of the visual field, blepharospasm, phlyctenular ophthalmia, conjunctivitis, keratitis, mydriasis-Goris and Trousscau-amaurosis, amblyopia, strabismus and even glaucoma, myopia-congestion of ureal tract (Iandolt) and astigmatism (spasmodic).

The supposed nasal and nasopharyngeal causes were as numerous as the resulting eye troubles, among them being acute rhinitis, chronic hypertrophic rhinitis, synechiæ, nasal polyps, growths, diseases of the accessory sinuses, adenoid tissue, enlarged tonsils, simple atrophic rhinitis, ozena and nasal hydrorrhea.

Coryza gives rise to lachrymation, conjunctival injection, dacryocystitis and ocular pains. Chronic rhinitis produces rebellious conjunctivitis and blepharitis, phlyctenular conjunctivitis, epiphora-asthenopia-myopia (?) - congestion of uveal tract (Landolt) - and astigmatism (spasmodic). Ozena produces ulcers of the cornea, dacryocystitis, neuritis, iritis, chorioretinitis, amblyopia, amaurosis and orbital phlegmon. Purulent rhinitis causes iritis and uveitis, phlebitis of ophthalmic veins, and contraction of the visual field. Sinusitis-frontal, maxillary, ethmoidal, etc--produces orbital neuralgia, iritis, iridochoroiditis, orbital cellulitis, neuritis, exophthalmus, diplopia, etc. To nasal hydrorrhea is due neuritis and atrophy of the optic nerve. Nasal synechiæ cause blepharospasm, conjunctivitis, amblyopia, and strabismus, and nasal diphtheria and croup, similar af-

* Presented to the Section on Ophthalmology, at the Fiftieth Annual Meeting of the American Medical Association, held at Columbus, Ohio, June 6-9, 1899. 